\title{
Retinal changes in Alzheimer's disease: Disease Mechanisms to Evaluation perspectives
}

M Mirzaei ${ }^{1,2,3 *}$, VB Gupta ${ }^{4}$, VK Gupta ${ }^{2}$

Department of Molecular Sciences, Macquarie University, Sydney, NSW, Australia

${ }^{2}$ Department of Clinical Medicine, Macquarie University, Sydney, NSW, Australia

${ }^{3}$ Australian Proteome Analysis Facility, Macquarie University, Sydney, NSW, Australia

${ }^{4}$ School of Medical Sciences, Edith Cowan University, Joondalup, WA, Australia

\section{Article Info}

\section{Article Notes}

Received: March 02, 2018

Accepted: March 29, 2018

\section{${ }^{*}$ Correspondence:}

Dr. Mehdi Mirzaei

Department of Molecular Sciences, Macquarie University,

Sydney, NSW, Australia, Email:

mehdi.mirzaei@mq.edu.au

(c) 2018 Mirzaei M. This article is distributed under the terms of the Creative Commons Attribution 4.0 International License
For the past decade the concept of "the eye as a window to brain" is increasingly being explored to understand brain functioning in health and disease conditions. During embryonic development, the retina and optic nerve project from the telencephalic and hypothalamic fields, and are thus considered part of the central nervous system (CNS) ${ }^{1,2}$. Retinal tissue demonstrates multiple similarities with the brain tissue at the cellular and biochemical levels. Further, similar to the blood-brain barrier, retinal tissue maintains a distinct immune environment that is facilitated by the blood-retinal barrier ${ }^{3}$. Several endogenous mechanisms have been identified in the retina that protect the tissue from nonspecific damage linked with the immune responses ${ }^{4,5}$. A distinctive milieu comprising of glial cells, surface molecules and cytokines imparts an ability to perform specialized immune responses similar to that observed in the brain and spinal cord tissues ${ }^{6}$. The limitations of brain imaging such as capturing fluorescence signals through the skull, invasive nature of accessing CSF fluid and high costs involved in MRI and PET scans necessitate exploring alternative options and in this aspect retinal imaging may serve as surrogate marker of monitoring various brain diseases and understand their effects on the eyes.

With the advances in optical coherence tomography (OCT) and OCT angiography for the retina, both animal and human retinas can be assessed for experimental or disease associated differences in vivo. Retina is increasingly being investigated to study the effects of predominantly brain related pathological conditions such Alzheimer's disease $(\mathrm{AD})^{7}$, Parkinson's disease $^{8}$, Multiple sclerosis ${ }^{9}$ and most recently Amyotrophic lateral sclerosis (ALS) ${ }^{10}$.

Extracellular deposits and aggregates containing amyloid $\beta(A \beta)$ and intracellular neurofibrillary tangles composed of hyper-phosphorylated tau have been observed in the retina in animal models of $\mathrm{AD}^{7,11}$. Partial overlap of such molecular changes has also been reported in retinal neurodegenerative disorders such as age related macular degeneration (AMD) and glaucoma conditions ${ }^{12-15}$.

Clinically, AD patients exhibit a progressive decline in memory and impaired cognition and learning abilities, which are accompanied by visual and ocular manifestations, such as impaired pupil response, contrast sensitivity, thinning of retinal layers and decreased visual function ${ }^{13,16}$. Studies also suggest an increased incidence of glaucoma pathology in $\mathrm{AD}$ patients, which indicates a genetic or pathological link between the two disorders that either predisposes AD subjects 
to glaucoma or exacerbates the glaucoma pathology ${ }^{17,18}$. Another possibility is the manifestation of AD effects in a way that overlaps with glaucoma associated pathological changes in the retina and optic nerve. Detailed clinical and animal studies in pre-clinical stages and post-AD development stages will help to elucidate the similarities and differences in terms of ocular effects between the two disorders and understand the extent of overlap. A link between the two diseases is also suggested because both the diseases affect predominantly the inner retinal function and structure. Detailed analysis of the imaging changes in central and peripheral regions of the retina along with clinical assessment will help unravel the differential effects of the two disorders on the retina and optic nerve.

Studies in AD patients and animal models of AD such as in APP/PS1 and TG2576 mice models demonstrate retinal nerve fibre layer thinning and loss of the retinal ganglion cells (RGCs), which is also a hallmark of glaucoma $^{7,19}$. Furthermore, increased $A \beta$ levels and tau hyperphosphorylation has been identified in both $\mathrm{AD}$ as well as in glaucoma retinas ${ }^{20}$. Targeting $A \beta$ peptide using specific antibodies in glaucoma mice protected against the retinal ganglion cell loss and apoptosis, indicating a pathological role of this protein ${ }^{21}$. Awareness of the $A \beta$ accumulation in the retinas of $\mathrm{AD}$ subjects and $\mathrm{AD}$ animal models has led to an increasing body of work exploring biochemical mechanisms and a prospective biomarker role for retinal changes in AD.

Future studies will need to identify retinal changes that are specifically associated with AD pathology and can be effectively distinguished from the retinal deficits observed in glaucoma, AMD and ageing. Potential retinal changes if identified in early stages of AD may play a critical role in improving the disease diagnosis. In more established cases of $\mathrm{AD}$, ocular deficits may be used for non-invasive monitoring of the disease. For example, OCT imaging has revealed reduced peripapillary RNFL thickness in $\mathrm{AD}$ patients, with thinning of average, superior and inferior GCL compared to the control subjects ${ }^{22-24}$. Further, detailed analysis of macular quadrants has indicated significantly reduced macularvolume as well as loss of the nasal, temporal and inferior region thickness in $\mathrm{AD}^{25,26}$. Retinal imaging studies in larger cohorts of healthy ageing and potential $\mathrm{AD}$ subjects demonstrating mild cognitive impairment (MCI) will be helpful to identify biological markers for early diagnosis of the disease and for monitoring the disease progression. Changes in RNFL thickness have previously been reported in MCI subjects by different groups ${ }^{27,28}$. The molecular changes in the retina and optic nerve at different stages of AD will also provide us useful clues about the way various proteins and biochemical pathways are affected in various stages of disease. This information will help in targeted discovery of alterations in various proteins and molecular networks as a marker of the disease in accessible body fluids. These changes could be associated with either protein expression changes or post-translational modifications, which could then affect various cellular signalling pathways. It is important to highlight that these changes may show a direct correlation with brain pathology linked with AD or may be independent of these changes, but regardless will help to understand the novel mechanisms at the fore-front of $\mathrm{AD}$ pathology. Further, every case of $\mathrm{AD}$ is different and there may be significant variations between sporadic versus familial types of $\mathrm{AD}$ as well as with vascular dementias. We may also hope to expect significant differences in ocular effects of the disease with respect to sex, age and ethnicities. As AD is associated with a wide range of clinical presentationswith the technological advancements neuroscientists and neurologists could truly hope to develop a spectrum of retinal changes connected to $\mathrm{AD}$ in the near future.

\section{References}

1. Gupta V. One protein, multiple pathologies: multifaceted involvement of amyloid beta in neurodegenerative disorders of the brain and retina. Cell Mol Life Sci. 2016; 73: 4279-4297. doi:10.1007/s00018016-2295-x.

2. Cooper HM, Mick G, Magnin M. Retinal projection to mammalian telencephalon. Brain Res. 1989; 477: 350-357.

3. Campbell M, Humphries P. The blood-retina barrier: tight junctions and barrier modulation. Adv Exp Med Biol. 2012; 763: 70-84.

4. McPherson SW, Yang J, Chan CC, et al. Resting CD8 T cells recognize beta-galactosidase expressed in the immune-privileged retina and mediate autoimmune disease when activated. Immunology. 2003; 110: 386-396.

5. Streilein JW, Ma N, Wenkel H, et al. Immunobiology and privilege of neuronal retina and pigment epithelium transplants. Vision Res. 2002; 42: 487-495.

6. Kumar A, Pandey RK, Miller LJ, et al. Muller glia in retinal innate immunity: a perspective on their roles in endophthalmitis. Crit Rev Immunol. 2013; 33: 119-135.

7. Gupta VK. Amyloid beta accumulation and Inner retinal degenerative changes in Alzheimer's disease transgenic mouse. Neurosci Lett. 2016. doi:10.1016/j.neulet.2016.04.059.

8. Yu JG. Retinal nerve fiber layer thickness changes in Parkinson disease: a meta-analysis. PLoS One. 2014; 9: e85718, doi:10.1371/ journal.pone.0085718.

9. Cennamo G. Anatomical and functional retinal changes in multiple sclerosis. Eye (Lond). 2016; 30: 456-462. doi:10.1038/eye.2015.256.

10. Mukherjee N, McBurney-Lin S, Kuo A, et al. Retinal thinning in amyotrophic lateral sclerosis patients without ophthalmic disease. PLoS One. 2017; 12: e0185242, doi:10.1371/journal.pone.0185242.

11. Chiasseu M. Tau accumulation in the retina promotes early neuronal dysfunction and precedes brain pathology in a mouse model of Alzheimer's disease. Molecular neurodegeneration. 2017; 12: 58, doi:10.1186/s13024-017-0199-3.

12. Sivak,JM. The aging eye: common degenerative mechanisms between the Alzheimer's brain and retinal disease. Invest Ophthalmol Vis Sci. 2013; 54: 871-880, doi:10.1167/iovs.12-10827.

13. Javaid FZ, Brenton J, Guo L, et al. Visual and Ocular Manifestations of Alzheimer's Disease and Their Use as Biomarkers for Diagnosis 
and Progression. Front Neurol. 2016; 7: 55, doi:10.3389/ fneur.2016.00055.

14. Gupta VK, Rajala A, Rajala RV. Insulin receptor regulates photoreceptor CNG channel activity. Am J Physiol Endocrinol Metab. 2012; 303: E1363-1372, doi:10.1152/ajpendo.00199.2012.

15. Mirzaei M. Age-related neurodegenerative disease associated pathways identified in retinal and vitreous proteome from human glaucoma eyes. Scientific Reports. 2017; 7: 12685, doi:10.1038/ s41598-017-12858-7.

16. Hart NJ, Koronyo Y, Black KL, et al. Ocular indicators of Alzheimer's: exploring disease in the retina. Acta Neuropathol. 2016; 132: 767787, doi:10.1007/s00401-016-1613-6.

17. Bayer AU, Ferrari F. Severe progression of glaucomatous optic neuropathy in patients with Alzheimer's disease. Eye (Lond). 2002; 16: 209-212, doi:10.1038/sj/EYE/6700034.

18. Valenti DA. Alzheimer's disease and glaucoma: imaging the biomarkers of neurodegenerative disease. Int J Alzheimers Dis. 2011; 2010: 793931, doi:10.4061/2010/793931.

19. Chiu K. Neurodegeneration of the retina in mouse models of Alzheimer's disease: what can we learn from the retina. Age (Dordr). 2012; 34: 633-649, doi:10.1007/s11357-011-9260-2.

20. Gupta V. BDNF impairment is associated with age-related changes in the inner retina and exacerbates experimental glaucoma. Biochim Biophys Acta. 2014; 1842: 1567-1578, doi:10.1016/j. bbadis.2014.05.026

21. Liu B. Amyloid-peptide vaccinations reduce $\{$ beta\}-amyloid plaques but exacerbate vascular deposition and inflammation in the retina of Alzheimer's transgenic mice. Am J Pathol. 2009; 175: 2099-2110, doi:10.2353/ajpath.2009.090159.

22. Bayhan HA, Aslan Bayhan S, Celikbilek A, et al. Evaluation of the chorioretinal thickness changes in Alzheimer's disease using spectraldomain optical coherence tomography. Clin Exp Ophthalmol. 2015; 43: 145-151, doi:10.1111/ceo.12386.

23. Cunha LP, Almeida AL, Costa-Cunha LV,et al. The role of optical coherence tomography in Alzheimer's disease. Int J Retina Vitreous. 2016; 2: 24, doi:10.1186/s40942-016-0049-4.

24. Marziani E. Evaluation of retinal nerve fiber layer and ganglion cell layer thickness in Alzheimer's disease using spectral-domain optical coherence tomography. Invest Ophthalmol Vis Sci. 2013; 54: 59535958, doi:10.1167/iovs.13-12046.

25. Iseri PK, Altinas $\mathrm{O}$, Tokay $\mathrm{T}$, et al. Relationship between cognitive impairment and retinal morphological and visual functional abnormalities in Alzheimer disease. J Neuroophthalmol. 2006; 26: 18-24, doi:10.1097/01.wno.0000204645.56873.26.

26. Moschos MM Markopoulos I, Chatziralli I, et al. Structural and functional impairment of the retina and optic nerve in Alzheimer's disease. Curr Alzheimer Res. 2012; 9: 782-788.

27. Oktem EO, Derle E, Kibaroglu S, et al. The relationship between the degree of cognitive impairment and retinal nerve fiber layer thickness. Neurol Sci. 2015; 36: 1141-1146, doi:10.1007/s10072-014-2055-3.

28. Ascaso FJ, Cruz N, Modrego PJ, et al. Retinal alterations in mild cognitive impairment and Alzheimer's disease: an optical coherence tomography study. J Neurol. 2014; 261: 1522-1530, doi:10.1007/ s00415-014-7374-z. 